\title{
基于主成分的大坝观测数据多效应量统计分析 研究
}

虞鸿 $^{(12 *}$, 吴中如 ${ }^{(1)}$, 包腾飞 ${ }^{(1)}$, 张岗 ${ }^{\text {(1) (2) }}$

(1) 河海大学水文水资源与水利工程科学国家重点实验室, 南京 210098 ;

(2) 河海大学水资源高效利用与工程安全国家工程研究中心, 南京 210098

*E-mail: fishred2006@yahoo.com.cn

收稿日期: 2009-07-03; 接受日期: 2009-09-02

国家自然科学基金项目(批准号：50909041，50539010，50539030，50539110，50809025，50879024)、国家科技支撑计划课题(批准号： 2006BAC14B03, 2008BAB29B03)、中国水电工程顾问集团公司科技项目(批准号: CHC-KJ-2007-02)和河海大学自然科学基金项目(批准号: 2008426811)资助

摘要 传统的单效应量分析在处理具有多重共线性的大坝监测数据时有较大的局限性, 本 文引入主成分分析方法对相关的多效应量进行重构, 探讨确定主成分的方法, 提取影响数据 变异的少数主成分. 对于稳定的观测数据建立基于主成分的多效应量观测值整体控制域; 对 于有趋势性变化的观测数据，在建立主成分统计预报模型的基础上建立了未来观测值的控制 域. 通过工程实例证明, 利用主成分分析方法进行多效应量分析可以实现数据缩减, 减少数

关键词

大坝安全监控

多效应量

主成分分析

数据缩减 据冗余, 降低噪声和虚假报警率, 能够进行高效地数据分析, 具有广阔的应用前景.

几个世纪以来, 水利工程作为构成社会整体所 不可或缺的一种基础设施, 为人类生存和经济社会 持续发展提供防洪、供水保障, 并产生了通航、发电、 养殖、灌溉等综合效益. 在工程界, 水库大坝的失事 公认为是一种“小概率, 大损失”的灾难事件. 尤其是 当大坝长期运行以后(一般 50 年及以上), 由于设计 时较低的标准和建设时尚未成熟的施工工艺, 大多 数工程出现结构老化或设计泄洪能力不足等缺陷, 导致大坝失事的风险逐年增加 ${ }^{[1]}$; 如果遭遇极端的荷 载如特大洪水、地震等, 一旦工程失事, 将直接威胁 到工程下游经济社会和人员生命的安全.

大坝性能监测是大坝安全计划的重要组成部份, 主要采用两种方式: 1)周期性的巡视检查和 2)处理分 析监测仪器测得的数据. 其中后者采集的数据能够
及时反映大坝结构和性能的重要变化, 已经成为我 国水利工程安全监控规范的重要组成部分 ${ }^{[2]}$. 近年来, 制约国内大坝安全定检工作的主要瓶颈已经从原来 的缺少实时数据转变为数据分析出现较大延迟, 这 种转变的主要原因是在数据分析方法的研究和创新 上投入的精力和在提高大坝性能数据采集能力方面 做出的努力不成比例. 实际表现为以下悖论: 数据自 动采集系统(automatic data acquisition systems, ADAS) 发展迅速, 新的监测方法和仪器不断涌现并投入使 用, 采集频率高, 数据量日益增大; 而数据统计分析 方法还处于 “一点一模型”单效应量分析阶段，对于 物理系统中普遍存在的噪声和异常数据仍需要专家 凭经验进行认定和处理, 分析效率受到较大限制. 虽 然先进的数据采集方式提供的大量翔实的即时实测

引用格式: Yu H, Wu Z R, Bao T F, et al. Multivariate analysis in dam monitoring data with PCA. Sci China Tech Sci, 2010, 53: 1088-1097, doi: 10.1007/s11431-010-0060-1 
数据, 但骤增的分析工作量直接导致分析用时的几 何增长. 由于分析的低效导致补救措施延误, 错过最 佳时机, 不仅会增加修补工程的成本, 更为严重的是 应急措施不力可能引起工程失事, 造成不可挽回的损 失. Dibiagio 在 20 届世界大坝委员会的报告中强调需 要开发高效的分析工具用于大坝性能的评估 ${ }^{[3]}$.

为了提高数据分析效率, 压缩冗余数据, 减小测 量数据中噪声的影响, 实现数据缩减, 需要采用一种 新的统计分析方法: 主成分分析(principal component analysis). 主成分分析是在保证数据信息丢失最少的 原则下, 对高维变量空间进行降维处理. 历史上有许 多成功运用 PCA 的实例, 如 Stone 在研究美国国民经 济都用主成分取代原有变量的方法, 且能保证 $95 \%$ 以上的分析精度 ${ }^{[4]}$. 在大坝监测资料分析方面, Behrouz 等人在 Idukki 拱坝, Daniel Johnson 连拱坝, Chute-à-Caron 重力坝的位移、应力和渗流分析中使 用了主成分分析的方法, 并结合 Hydrostatic-SeasonTime(HST)模型进行效应量参数估计, 采用少量的主 成分概括大坝的性态, 获得了良好的分析效果 ${ }^{[5]}$. 李 雪红等人利用主成分分析加强神经网络的学习能力 进而处理大坝观测数据的多重共线性问题 ${ }^{[6]}$. 刘成栋 采用主成分分析方法提取数据信息, 进行影响大坝 的多因素赋权 ${ }^{[7]}$. 陈龙建立了基于主成分的碾压混凝 土坝层面性态模糊综合评判模型 ${ }^{[8]}$. 作者将该方法运 用于大坝安全监控数据分析, 研究基于主成分的置 信椭圆用于大坝安全观测数据的分析和预警.

\section{1 虚假警报、数据缩减和消除噪声}

虚假警报、数据冗余和噪声影响是大坝安全监测 数据中普遍存在的三个主要问题, 下面逐一进行分析.

\section{1 虚假警报}

所谓虚假警报是指由于某种偶然因素仪器失去 控制, 测得的数据出现较大误差而失真; 分析这些数 据可能得出一些不利的结论而发出警报, 但现实上 工程仍处于安全的状态. 从监测系统布置的角度来 看, 大部分反映大坝性态的特征和影响大坝运行的 因素都是用仪器监控的, 如: 1)位移, 2)孔隙压力和扬 压力, 3)水位和流量, 4)渗流, 5)水质, 6)温度, 7)接缝 与裂缝的尺寸, 8)地震活动, 9)降雨, 10)应力和 11)应 变. 传统的单效应量分析最终成果是取显著性水平 $\alpha=0.05$, 得到一个 $100(1-\alpha) \%$ 的观测值预报区间并
据此进行监测和报警:

$$
\begin{gathered}
\hat{y}\left(x_{0}\right)-t_{\frac{\alpha}{2}, n-p}\left(\sqrt{S_{y, x}^{2}\left(1+x_{0}^{\prime}\left(\boldsymbol{X}^{\mathrm{T}} \boldsymbol{X}\right)^{-1} x_{0}\right)}\right) \leq y_{0} \\
\leq \hat{y}\left(x_{0}\right)+t_{\frac{\alpha}{2}, n-p}\left(\sqrt{S_{y, x}^{2}\left(1+x_{0}^{\prime}\left(\boldsymbol{X}^{\mathrm{T}} \boldsymbol{X}\right)^{-1} x_{0}\right)}\right), \\
S_{y, x}=\sqrt{\frac{\hat{\boldsymbol{Y}} \hat{\boldsymbol{Y}}-\boldsymbol{b}^{\prime} \boldsymbol{X}^{\prime} \boldsymbol{Y}}{n-p-1}},
\end{gathered}
$$

其中 $n$ 为观测样本容量, $p$ 为预测变量(因子)规模, $\boldsymbol{b}$ 为回归系数向量, $x_{0}$ 为预报变量组成的向量, $\hat{y}$ 为观 测值的预测值, $\boldsymbol{X}$ 为预测变量的矩阵 $(n \times p), \boldsymbol{Y}$ 为某一 效应量观测值矩阵, $\hat{\boldsymbol{Y}}$ 为回归分析得到的样本估计 值矩阵, $S_{y, x}$ 为估计标准差.

根据上述结论可以对由测量误差导致的虚假警 报进行概率分析. 从目前的资料分析实践来看，监测 大坝性态的各个效应量都可作为随机变量, 为了简 化分析, 假定这些变量都相互独立, 不考虑它们之间 可能存在的相关性, 根据中心极限定理, 各个效应量 长期观测序列中的观测值标准化后服从标准正态分 布, 取显著性水平 $\alpha=0.05$ 的双侧分位点, 观测值处 于预报区间的概率为

$$
\begin{aligned}
P(A) & =P\left\{y_{0} \mid \hat{y}\left(x_{0}\right)-2.575 \sigma \leq y_{0} \leq \hat{y}\left(x_{0}\right)+2.575 \sigma\right\} \\
& =0.99 .
\end{aligned}
$$

考虑到大坝失事是小概率事件, 异常值的出现 主要是测量误差导致的, 所以可以认为单个效应量 的虚假警报率近似等于

$$
\begin{aligned}
P(B)= & P\left\{y_{0} \mid y_{0}<y_{0}-2.575 \sigma\right\} \\
& +P\left\{y_{0} \mid y_{0}>y_{0}+2.575 \sigma\right\} \\
= & 1-P(A)=1-0.99=0.01 .
\end{aligned}
$$

对同时监测 $l$ 个效应量的水利工程而言, 若不考 虑项目间的相关关系, 则对所有项目进行一次观测 而无警情报告的概率为

$$
P(C)=(1-P(B))^{l}=0.99^{l} .
$$

绘制虚假警报率与监测效应量规模的关系曲线 (图 1), 可以发现当工程的规模达到一定程度以上时 (l>200)，虚假警报率已经超过 90\%，规模达到 400 时， 虚假警报率已经近似等于 1 . 而监测实践表明，一般 监测条件下的中型水利枢纽, 布置的仪器数量也有 数百个. 至于三峡大坝这样大型的工程, 监测系统设 计需布置的仪器超过 8000 支, 某些仪器还能监测多 


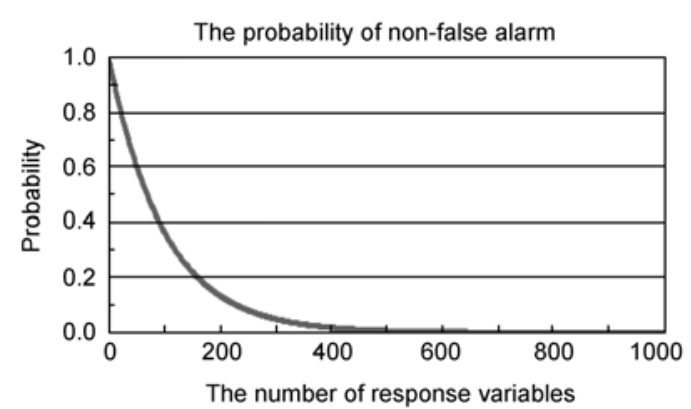

图 1 单次观测无报警的概率曲线

个效应量，这些情况将进一步提高虚假警报率.

可以说频繁的虚假警报是制约传统分析方法大 规模应用的瓶颈, 但这种情况不是不可避免的. 近年 来大坝安全监测的实践中发现，许多效应量之间存 在一定的相关关系, 如: 同一坝段各高程倒垂的测量 值之间、不同坝段引张线的测量值之间等项目都具有 明显的相关关系. 利用这些相关性引入主成分分析 方法可以进行双变量分析或多变量分析, 用主成分 的置信椭圆对未来观测值进行判断. 这样一方面用 主成分代替效应量减小了变量的规模，另一方面减 小了测量误差的影响，从而降低了虚假警报率.

\section{2 数据缩减}

上述分析中涉及到了多种效应量之间的相关关 系，不同的效应量观测数据往往是大坝同一性态的 反映, 从这个意义上讲, 观测数据中广泛存在着一定 程度上的数据冗余. 在 ADAS 技术成熟之前, 人工数 据采样的间隔很难保持一致, 为了进行多变量分析通 常需要大量的内插和外推计算, 工作量大且难以保证 精度, 因此这一时期多元分析方法受到较大的局限. 而通常采用的传统单效应量分析方法又割裂了分析 目标之间的相关关系, 保留了较多的几余数据, 直接 影响了分析的效率. 随着自动化程度的提高, 带来了 两方面的影响, 不利的一面是庞大的采集数据量导致 越来越大的分析延迟; 有利的一面是自动化技术能够 保证多个仪器以相同的时间间隔测量, 自动满足双变 量分析或多变量分析对数据序列的要求.

主成分分析方法从海量的监测数据中集中信息, 消除冗余. 同时通过提取主成分实现数据缩减, 缩短 获得分析成果的延迟时间.

\section{3 消除噪声}

大坝安全监测数据分析的首要问题是确认测量
数据的准确性和可靠性, 但是再精确的测量方法总 还是存在一定程度的测量误差. 测量误差可分为两 类: 1)系统误差; 2)随机误差. 前者主要表现是测量值 总是大于 (或小于)真实值一个固定的程度(百分比或 定值), 可以通过重新配置监测系统而妥善解决; 后 者是测量中不可预见的偏差，可能来自于气流运动， 温度、相对湿度的变化, 电压的波动, 电磁的相互作 用等等，具体表现为测量值在真实值附近的摄动. 通 常随机误差可以用声音信号中的背景噪声来类比, 在数学上利用主成分分析方法分析长期观测的数据 序列, 有效地降低噪声, 可操作性强.

\section{2 主成分分析}

上述分析介绍了引入主成分分析的原因和目的, 该方法的基本思想是在不损失或很少损失原有信息 的前提下，将原来众多具有一定相关性的变量重新 组合成新的少数几个相互无关的综合变量，来代替 原来变量，这些新的综合变量称之为主成分.

\section{1 主成分分析原理}

假设有 $m$ 个效应量, 每个效应量有 $n$ 个观测值, 记为 $\left(x_{1}, x_{2}, \cdots, x_{m}\right)$, 为简单起见, 可以设 $x_{i}$ 的均值为 0 , 方差为 $1,1 \leqslant i \leqslant m)$, 构成一个 $n \times m$ 的矩阵:

$$
\boldsymbol{X}=\left[\begin{array}{cccc}
x_{11} & x_{12} & \cdots & x_{1 m} \\
x_{21} & x_{22} & \cdots & x_{2 m} \\
\vdots & \vdots & \ddots & \vdots \\
x_{n 1} & x_{n 2} & \cdots & x_{n m}
\end{array}\right] .
$$

主成分分析的目的是在于用 $m$ 个原始变量 $\left(x_{1}, x_{2}\right.$, $\left.\cdots, x_{m}\right)$ 构造少数几个新的综合变量, 使新变量为原 始变量的线性组合，且新变量互不相关. 新的变量包 含 $m$ 个原始变量的绝大部分的信息. 不妨定义 $z_{1}, z_{2}$, $\cdots, z_{p}$ 为新的综合变量, 其中每一个新的综合变量都 是 $m$ 个原始变量的线性组合:

$$
\left\{\begin{array}{c}
z_{1}=l_{11} x_{1}+l_{12} x_{2}+\cdots+l_{1 m} x_{m}=\sum_{i=1}^{m} l_{1 i} x_{i}, \\
z_{2}=l_{21} x_{1}+l_{22} x_{2}+\cdots+l_{2 m} x_{m}=\sum_{i=1}^{m} l_{2 i} x_{i}, \\
\vdots \\
z_{p}=l_{n 1} x_{1}+l_{n 2} x_{2}+\cdots+l_{n m} x_{m}=\sum_{i=1}^{m} l_{n i} x_{i} .
\end{array}\right.
$$

同时满足下列几个条件: 
1) $z_{i}$ 与 $z_{j}(i \neq j ; i, j=1,2, \cdots, p)$ 相互无关;

2) $z_{1}$ 是 $x_{1}, x_{2}, \cdots, x_{m}$ 的一切线性组合中方差最大 者; $z_{2}$ 是与 $z_{1}$ 不相关的 $x_{1}, x_{2}, \cdots, x_{m}$ 的所有线性组合中 方差最大者; $\cdots z_{p}$ 是与 $z_{1}, z_{2}, \cdots, z_{p-1}$ 都不相关的所有 线性组合中方差最大者.

则新变量 $z_{1}, z_{2}, \cdots, z_{p}$ 分别称为原变量 $x_{1}, x_{2}, \cdots$, $x_{m}$ 的第一, 第二, $\cdots$, 第 $p$ 主成分.

以上的分析可以看出, 主成分分析的实质就是 确定原来变量 $x_{j}(j=1,2, \cdots, m)$ 在诸主成分 $z_{i}(i=1,2, \cdots$, $p)$ 上的系数 $l_{i j}(i=1,2, \cdots, p, j=1,2, \cdots, m)$. 从数学上可 以证明, 它们分别是 $m$ 个原始变量 $\left(x_{1}, x_{2}, \cdots, x_{m}\right)$ 相关 矩阵的前 $p$ 个具有较大特征值所对应的特征向量, 而 各个综合变量 $z_{i}$ 的方差 $\operatorname{var}\left(z_{i}\right)$ 恰好是相应的特征根 $\lambda_{i}$. 各主成分的方差贡献大小按特征值顺序排列, 是依 次递减的, 即

$$
\lambda_{1} \geq \lambda_{2} \geq \cdots \geq \lambda_{p} \geq 0 .
$$

\section{2 主成分分析基本步骤}

\subsection{1 计算相关矩阵的特征值}

PCA 是一种应用于相关系数矩阵 $(\boldsymbol{R})$ 或协相关矩 阵 $(S)$ 的统计分析技术. 寻找主成分 PC 的第一步是计 算(协)相关矩阵的特征值:

$$
(\boldsymbol{R}-\lambda \boldsymbol{I}) p=0,
$$

其中 $\boldsymbol{I}$ 是 $m$ 阶的单位阵. 有许多不同的数值算法可以 用于计算特征向量及其对应的特征值 ${ }^{[9]}$. 解得的特征 值 $\lambda_{j}(j=1,2, \cdots, m)$ 可以构成一个对角矩阵 $\boldsymbol{\Lambda}$, 它们对 应的特征向量的集合可记为 $\boldsymbol{p}_{j}(j=1,2, \cdots, m)$. PC 是 所有变量的线性组合, 其中组成主成分的各个变量 的权重由特征向量的各个分量决定. 通过新构建的 主成份所能解释原始数据的变异程度可以用下面两 式计算:

$$
\begin{aligned}
& \frac{\lambda_{k}}{\sum_{j=1}^{m} \lambda_{j}} \times 100 \%, \\
& \frac{\sum_{j=1}^{k} \lambda_{j}}{\sum_{j=1}^{m} \lambda_{j}} \times 100 \%,
\end{aligned}
$$

(10)式为第 $k$ 个主成分对原始数据变化的影响程度, 称为贡献率; (11)式可以表示前 $k$ 个主成分对原始数
据变化的总影响程度, 称为累积贡献率.

\subsection{2 确定主成分的个数}

可以由以下几种方法确定.

1) 累积贡献率. 先将特征值按从大到小排序, 按主特征向量构成的主成分即为第一主成分. 从第 一主成分开始逐个增加成分, 用(11)式计算相应的累 积贡献率, 重复上述步骤直到能较好的解释原始数 据变 化为止，一般需达到 70\% 90\%。

2) 平均特征值. 取所有特征值的平均数作为标 准，特征值大于平均特征值对应的特征向量就是所 需的主成分. 根据研究发现, 当原始变量的个数小于 30 而且他们之间存在高度的相关性时, 这种方法篮 选取出的主成分是精确的 ${ }^{[10]}$.

3) 作图法. 点绘特征值的碎石图, 当曲线变平 时，即为主成分与次要成分的分位点.

4) 信号噪声法. 主成分与次要成分之间的关系, 可以用信号与噪声的关系来解释. 相邻成分之间的 差异, 可以用下式比较:

$$
\gamma_{k}=\frac{\lambda_{k}}{\lambda_{k+1}},(k=1,2, \cdots, p-1) .
$$

当主成分识别完成时, $\gamma_{k}$ 表示的是最小主成分特 征值 $\lambda_{k}$ 与最大噪声信号特征值 $\gamma_{k+1}$ 的比值. 由于信号 与噪声的性质不同, 它们之间的比值远大于主成分 之间和噪声信号之间的比值，可以作为区分主成分 与次要成分的标志.

\subsection{3 构造主成分}

当 $k$ 个主成分识别完成后，原始数据可以用这些 主成分重构:

$$
\boldsymbol{Y}=\boldsymbol{Z}+\boldsymbol{E}=\boldsymbol{X} \boldsymbol{p}^{\prime}+\boldsymbol{E},
$$

其中 $\boldsymbol{E}$ 为残差矩阵, $\boldsymbol{Z}$ 为主成份矩阵, $\boldsymbol{p}$ 是前 $k$ 个主特 征向量矩阵 $\boldsymbol{p}=\left\{\boldsymbol{p}_{1}, \boldsymbol{p}_{2}, \cdots, \boldsymbol{p}_{k}\right\}$. 残差矩阵包含了数 据中用 PCA 无法解释的部分, 一部分是随机误差即 数据中的噪声信号, 因为随机误差总是与主成分不 相关, 另一部分是相当次要的成分对分析目标影响 不大.

\section{3 主成分安全监控方法}

如果大坝的性态稳定，那么主成分的过程线不 会出现随时间的趋势性变化. 对于这种稳定的观测 
序列, 未来的观测值可以通过建立主成分整体观察 值的控制域进行结构的安全监控; 如果大坝受到混 凝土蠕变, 干缩、碱骨料反应等因素的影响, 主成分 的过程线表现出随时间的趋势性变化. 这就需要建 立以模型预测值为中心的, 未来单个主成分观测值 的控制域进行结构安全监控. 下面介绍两种具体的 实施方法.

\subsection{1 整体观察值的控制域}

当大坝的性态保持稳定时, 利用收集到的数据 $x_{1}, x_{2}, \cdots, x_{n}$, 提取主分量 $z_{1}, z_{2}, \cdots, z_{p}$ 来确定某一个或 某些未来观测值的控制域. 若大坝性态稳定, 则观测 值可视为独立同分布，其分布服从 $N_{p}(u, \sigma)$.

设 $z_{1}, z_{2}, \cdots, z_{p}$ 独立同分布, 分布为 $N_{p}(u, \sigma)$, 记 $\boldsymbol{Z}$ 为来自相同分布的一个未来观测值, 此时, 统计量

$$
T^{2}=\frac{n}{n+1}(\boldsymbol{Z}-\overline{\boldsymbol{Z}})^{\prime} \boldsymbol{S}^{-1}(\boldsymbol{Z}-\overline{\boldsymbol{Z}}),
$$

其中

$$
\begin{aligned}
\boldsymbol{S} & =\left[\begin{array}{cccc}
S_{11} & S_{12} & \cdots & S_{1 p} \\
S_{21} & S_{22} & \cdots & S_{2 p} \\
\vdots & \vdots & \ddots & \vdots \\
S_{p 1} & S_{p 2} & \cdots & S_{p p}
\end{array}\right] \\
& =\left\{S_{i k}=\frac{1}{n-1} \sum_{j=1}^{n}\left(z_{j i}-\bar{z}_{i}\right)\left(z_{j k}-\bar{z}_{k}\right)\right\},
\end{aligned}
$$

服从分布 $\frac{(n-1) p}{n-p} F_{p, n-p}$, 且其 100(1- $\left.\alpha\right) \%$ 的 $p$ 维预测 椭球由满足如下不等式的全部 $z$ 决定:

$$
\frac{n}{n+1}(\boldsymbol{Z}-\overline{\boldsymbol{Z}})^{\prime} \boldsymbol{S}^{-1}(\boldsymbol{Z}-\overline{\boldsymbol{Z}}) \leq \frac{(n-1) p}{n-p} F_{\alpha}(p, n-p),
$$

它是以样本平均值 $\overline{\boldsymbol{Z}}$ 为中心的 $p$ 维椭球, 该椭球的相 关参数的确定如下.

由于 $\boldsymbol{S}$ 对称且正定，则由线性代数可知, $\boldsymbol{S}$ 有实 的特征值且均大于零. 不妨设 $p$ 个特征值为

$$
\lambda_{1} \geq \lambda_{2} \geq \cdots \geq \lambda_{p} \geq 0,
$$

其相应的单位特征向量为 $u_{1}, u_{2}, \cdots, u_{p}$, 它们均为 $p$ 维 列向量，且满足以下性质:

$$
\boldsymbol{u}_{i}^{\prime} \boldsymbol{u}_{j}= \begin{cases}1, & i=j, \\ 0, & i \neq j .\end{cases}
$$

记 $\boldsymbol{U}=\left(u_{1}, u_{2}, \cdots, u_{p}\right)^{\mathrm{T}}$ ，则 $\boldsymbol{U}$ 为正交阵，即

$$
\boldsymbol{U} \boldsymbol{U}^{\mathrm{T}}=\boldsymbol{U}^{\mathrm{T}} \boldsymbol{U}=\boldsymbol{I}
$$

记 $\boldsymbol{\Lambda}=\left[\begin{array}{llll}\lambda_{1} & & & \\ & \lambda_{2} & & \\ & & \ddots & \\ & & & \lambda_{p}\end{array}\right]$, 则 $\boldsymbol{S}^{-1}=\boldsymbol{U} \boldsymbol{\Lambda} \boldsymbol{U}^{\mathrm{T}}=\sum_{i=1}^{p} \frac{\boldsymbol{u}_{i} \boldsymbol{u}_{i}^{\mathrm{T}}}{\lambda_{i}}$,

则(16)式可以改写为

$$
\sum_{i=1}^{p} \frac{\frac{n(n-p)}{p\left(n^{2}-1\right)}(\boldsymbol{Z}-\overline{\boldsymbol{Z}})^{\prime} \boldsymbol{u}_{i} \boldsymbol{u}_{i}^{\mathrm{T}}(\boldsymbol{Z}-\overline{\boldsymbol{Z}})}{\lambda_{i}} \leq F_{\alpha}(p, n-p) .
$$

这样, 观测值的 $100(1-\alpha) \%$ 的置信域是以样本平均值

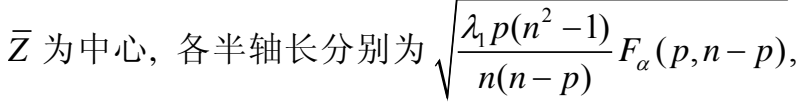
$\sqrt{\frac{\lambda_{2} p\left(n^{2}-1\right)}{n(n-p)} F_{\alpha}(p, n-p)}, \cdots, \sqrt{\frac{\lambda_{p} p\left(n^{2}-1\right)}{n(n-p)} F_{\alpha}(p, n-p)}$ 各轴的方向为 $\lambda_{i}$ 对应的特征向量 $\boldsymbol{u}_{i}, i=1,2, \cdots, p$.

特别的, 当 $p=2$ 时, $95 \%$ 的预测椭圆为

$$
(Z-\bar{Z})^{\prime} S^{-1}(Z-\bar{Z}) \leq \frac{2\left(n^{2}-1\right)}{n(n-2)} F_{0.05}(2, n-2),
$$

如果未来观测值 $\boldsymbol{Z}$ 落在控制椭圆之外，就记 $\boldsymbol{Z}$ 失控.

\subsection{2 未来单个观察值的控制域}

由于混凝土存在蠕变, 干缩, 碱骨料反应等, 混 凝土的性质随时间而变化. 而主成分可以通过 HST (Hydrostatic-Season-Time) 模型 ${ }^{[11]}$ 进行预测, 确定预 测值周围一随机区域，它包含观察值的概率为 $1-\alpha$.

HST 模型的主要公式如下:

$$
\begin{gathered}
D(t)=H(h)+S(\theta)+T(t), \\
H(h)=a_{1}+a_{2} h+a_{3} h^{2}+a_{4} h^{3}+a_{5} h^{4}, \\
S(\theta)=a_{6} \sin \theta+a_{7} \cos \theta+a_{8} \sin \theta \cos \theta+a_{9} \sin ^{2} \theta, \\
T(t)=c_{1} t^{\prime}+c_{2} t^{\prime 2}+c_{3} t^{\prime 3},
\end{gathered}
$$

其中 $D(t)$ 为效应量, $H(h)$ 为水压分量, $S(\theta)$ 为温度分量, $T(t)$ 为时效分量, $h=\frac{H-H_{\text {min }}}{H_{\text {max }}-H_{\text {min }}}, t^{\prime}=t-t_{0}, \theta=\frac{2 \pi t^{\prime}}{365}$, $H_{\text {min }}, H_{\text {max }}$ 是观测序列中相对的最高和最低库水位, $t_{0}$ 是统计分析收集的数据记录中的起始日期.

预测值的 $100(1-\alpha) \%$ 的置信域为 


$$
\frac{n(n-p)}{p(n-1)}(Z-\hat{Z})^{\prime} S^{-1}(Z-\hat{Z}) \leq F_{\alpha}(p, n-p) .
$$

它是以预测值 $\hat{Z}$ 为中心的 $p$ 维椭球, 该椭球的相关参 数的确定仿照(17) (19)式, 则(26)式可以改写为

$$
\sum_{i=1}^{p} \frac{\frac{n(n-p)}{p(n-1)}(Z-\hat{Z})^{\prime} \boldsymbol{u}_{i} \boldsymbol{u}_{i}^{\mathrm{T}}(Z-\hat{Z})}{\lambda_{i}} \leq F_{\alpha}(p, n-p) .
$$

这样, 观测值的 $100(1-\alpha) \%$ 的置信域是以预测值为中心, 各半轴长 $s a$ (semi-axes)分别为 $\sqrt{\frac{\lambda_{1} p(n-1)}{n(n-p)} F_{\alpha}(p, n-p)}$, $\sqrt{\frac{\lambda_{2} p(n-1)}{n(n-p)} F_{\alpha}(p, n-p)}, \cdots, \sqrt{\frac{\lambda_{p} p(n-1)}{n(n-p)} F_{\alpha}(p, n-p)}$. 各轴的方向为 $\lambda_{i}$ 对应的特征向量 $\boldsymbol{u}_{i}, i=1,2, \cdots, p$.

特别的, 当 $p=2$ 时, $95 \%$ 的预测椭圆为

$$
(\boldsymbol{Z}-\hat{\boldsymbol{Z}})^{\prime} \boldsymbol{S}^{-1}(\boldsymbol{Z}-\hat{\boldsymbol{Z}}) \leq \frac{2(n-1)}{n(n-2)} F_{2, n-2}(0.05) .
$$

如果不考虑预测值本身存在误差, 上述结论是 精确的, 但由于预测误差的不可避免, 需要综合考虑 其对半轴长的影响, 本文建议采用以下公式对半轴 长进行调整:

$$
s a=s a+\mu S,
$$

其中 $\mu$ 为折减系数, 与模型的相关系数有关, 一般为 0.4 0.5, $S$ 为模型的回归标准差. 折减过程需保证控 制椭圆的偏心率不变. 如果未来观测值 $\boldsymbol{Z}$ 落在控制椭 圆之外, 就记 $\boldsymbol{Z}$ 失控.

观测值中的粗差已作为次要成分在提取主成分 时已经剔除; 当大坝出现病变, 其监测值必然出现突 变, 对于上述两种控制域而言, 一旦失控, 立即报警.

\section{3 工程实例}

为了验证以上多效应量的分析方法, 现以陈村
水电站大坝为例, 应用上述方法进行主成分分析.

\section{1 工程概况}

陈村水电站位于晥南长江支流青七江上游, 是 一座以发电为主，兼顾防洪、灌溉、养殖、航运的综 合性水利枢纽, 其主要挡水建筑物为陈村重力拱坝. 该工程先后分三期施工, 由于浇筑二期混凝土时, 层 面上升速度较快, 浇筑层间歇时间短, 二期混凝土收 缩变形受到一期混凝土的强烈约束, 导致在一期混 凝土顶部(105 高程附近)产生裂缝. 裂缝长 300 余 m, 深 $5 \mathrm{~m}$ 以上, 削弱了坝体刚度, 并对坝体整体性产生 了影响, 裂缝原采用人工测缝计进行监测, 为了及时 观测裂缝开度变化情况, 从1998 年 8 月 17 日起, 分 别在 $5 \#, 8 \#, 18 \#, 26 \#$ 和 $28 \#$ 坝段新增了自动化测缝计 进行观测. 本文采用主成分分析的方法处理和分析 1998 年 8 月 17 日 2007 年 7 月 10 日自动化监测采集 的数据.

\section{2 构建主成分}

将 5 支测缝计的观测值作为原始变量, 记为 $x_{1}$, $x_{2}, \cdots, x_{5}$. 首先进行数据检查发现 1217 组数据中有 17 组共 33 个的数据出现仪器缺测, 占样本总数的 $1.4 \%$, 剔除出现数据异常的观测值组, 最后得到 1200 组观 测值. 绘制原始变量间的直方和散点矩阵图(图 2)并 计算相关系数矩阵 $\boldsymbol{R}$ (表 1$)$.

根据(9) (11)式计算 $\boldsymbol{R}$ 的特征值及其各自的贡献 率、累积贡献率和信号噪声比, 并绘制特征值的碎石 图, 见表 2 和图 3.

表 2 中 5 个特征值的平均值为 1.000 , 只有第一, 第二两个特征值大于 1 ; 结合图 2 我们发现数据变化 量的 $67.84 \%$ 可以由第一主成分解释, 第二主成分能 解释 $24.06 \%$, 两者的累积贡献度已经达到 $91.9 \%$, 已 经达到预定解释数据变化要求的精度; 从信号噪声

\section{表 1 相关系数表}

\begin{tabular}{cccccc}
\hline & $x_{1}$ & $x_{2}$ & $x_{3}$ & $x_{4}$ & 0.412 \\
$x_{1}$ & 1.000 & 0.215 & 0.424 & -0.434 \\
$x_{2}$ & 0.215 & 1.000 & -0.687 & 1.000 & 0.604 \\
$x_{3}$ & 0.424 & -0.687 & 0.948 & 0.620 \\
$x_{4}$ & 0.412 & -0.604 & 0.827 & 0.791 \\
$x_{5}$ & 0.434 & -0.620 & & 0.791 \\
\hline
\end{tabular}


表 $2 \boldsymbol{R}$ 的特征值及相关信息表

\begin{tabular}{ccccc}
\hline & $\lambda_{i}$ & 贡献率 & 累积贡献率 & $\lambda_{k} / \lambda_{k+1}$ \\
\hline 1 & 3.392 & 0.678 & 0.678 & 2.819 \\
2 & 1.203 & 0.241 & 0.919 & 4.929 \\
3 & 0.244 & 0.049 & 0.968 & 1.900 \\
4 & 0.129 & 0.026 & 0.994 & 4.016 \\
5 & 0.032 & 0.006 & 1.000 & 0.006 \\
\hline
\end{tabular}
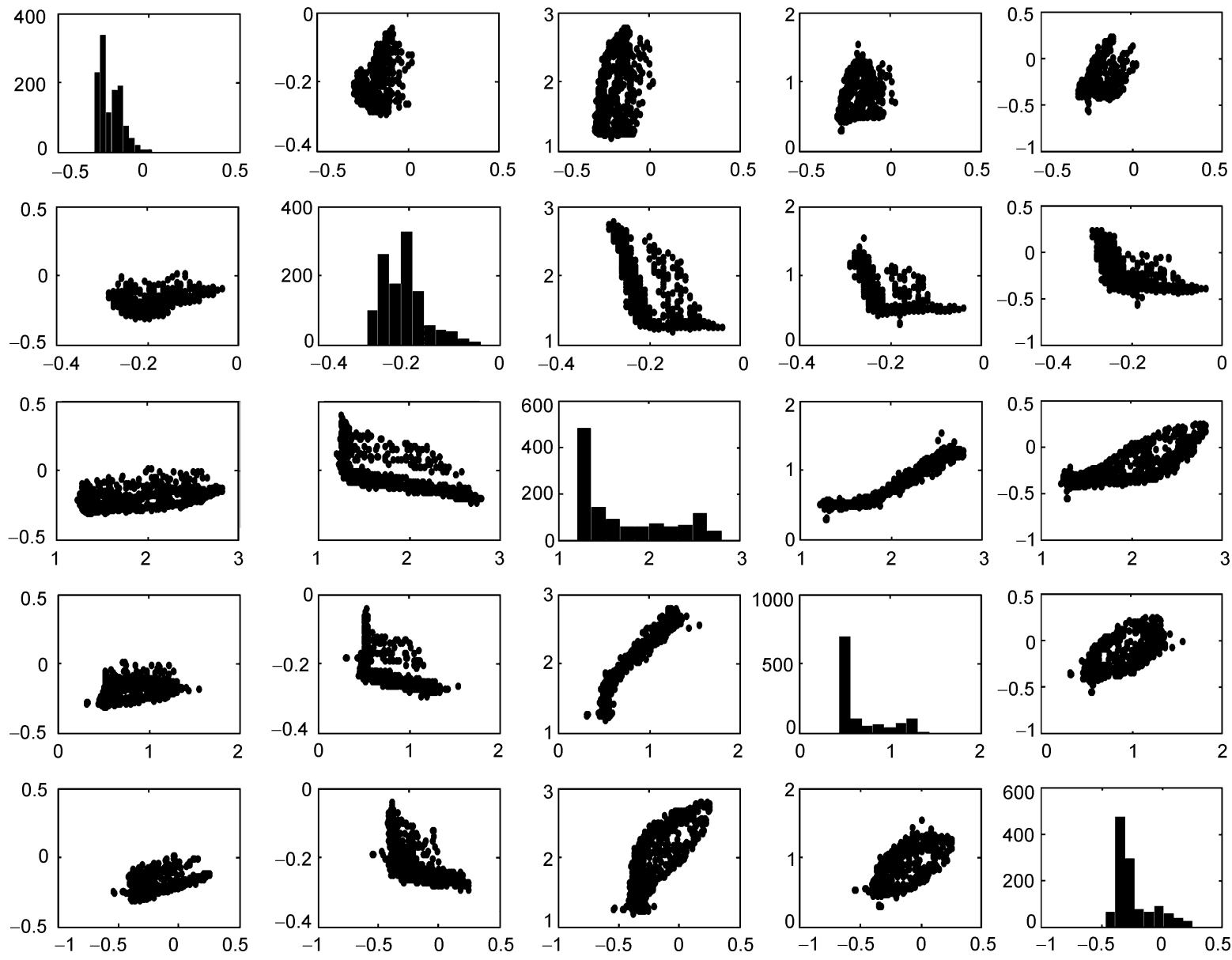

图 2 测点相关散点图和直方图矩阵

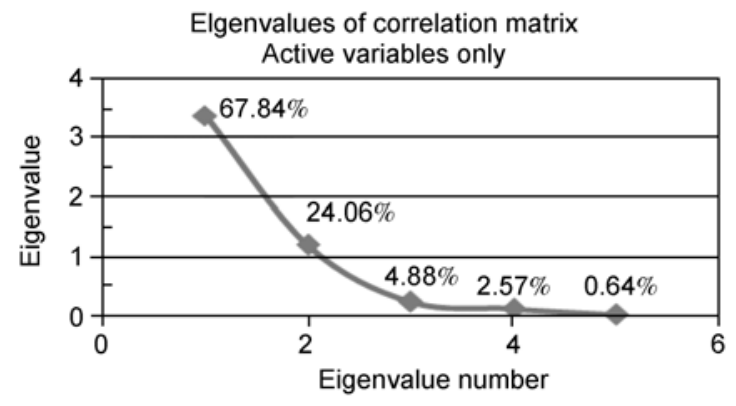

图 3 碎石图
比 $\left(\lambda_{k} / \lambda_{k+1}\right)$ 来看第二主成分与第三主成分之间的比例 最大, 所以可以认为第三主成分及以后的成分为噪 声. 主分量的载荷表见表 3 .

对载荷的分析表明，第一主成分主要解释 $x 18$, $x 26, x 28$ 的变化，第二主成分主要解释 $x 5, x 8$ 的变化. 提取主成分后将原来需要分析的五个效应量缩减为 两个, 达到了数据缩减的目的; 同时将 $8 \%$ 左右数据 变异考虑为噪声, 有效的减少了进行粗差调整的工 作量, 说明提取主成分的过程也是过滤噪声的过程. 
表 3 主成分载荷表

\begin{tabular}{ccc}
\hline \multirow{2}{*}{ 变量 } & \multicolumn{2}{c}{ 载荷 } \\
\cline { 2 - 3 } & PC1 & PC2 \\
\hline$x 5$ & -0.238 & -0.801 \\
$x 8$ & 0.390 & -0.596 \\
$x 18$ & -0.530 & -0.003 \\
$x 26$ & -0.514 & -0.036 \\
$x 28$ & -0.497 & -0.044 \\
\hline
\end{tabular}

\section{3 主成分监控方法和指标}

\subsection{1 建立整体观察值的控制域}

根据(14) (21)式，可得控制域方程为

$$
\begin{aligned}
& \left(Z-\left[\begin{array}{l}
1.181 \\
0.271
\end{array}\right]\right)^{\prime}\left[\begin{array}{ll}
0.224983 & -0.00898 \\
-0.00898 & 0.004166
\end{array}\right]^{-1}\left(Z-\left[\begin{array}{l}
1.181 \\
0.271
\end{array}\right]\right) \\
& \leq 6.010
\end{aligned}
$$

半长轴为 1.1644 , 方向为 $\boldsymbol{u}_{1}=\left[\begin{array}{lll}-0.99918 & 0.040546\end{array}\right]^{\mathrm{T}}$, 半短轴为 0.1512 , 方向为 $\boldsymbol{u}_{2}=[-0.99918-0.040546]^{\mathrm{T}}$. 整体散点图和控制域图如图 4 所示.

\subsection{2 建立未来观察值的控制域}

从整体观察值的散点图结合 PC1, PC2 和环境量 的过程线(图 5, 图 6 和图 7), 可以发现主成分的每年 的峰值存在增长的趋势, 这说明裂缝的性态尚未稳 定, 需要建立未来观察值的控制域对其进行监控. 而 且根据(22) (25)式建立 PC1 与 PC2 的 HST 预报模型, 模型的参数见表 4 .

PC1 的 HST 模型相关系数为 $R=0.973$, 标准差
$S=0.46, \mathrm{PC} 2$ 的 HST 模型相关系数为 $R=0.954$, 标准差 $S=0.082$.

PC1 与 PC2 的变化不同步, 不会同时达到峰值. 不利荷载组合并不仅仅存在于主成分出现峰值的时 刻, 所以确定 PC1 和 PC2 的联合控制域对于结构安 全有最要意义. 根据(26) (28)式, 可得控制域方程为

$\left(\boldsymbol{Z}-\left[\begin{array}{l}\hat{Z}_{1} \\ \hat{Z}_{2}\end{array}\right]\right)^{\prime}\left[\begin{array}{ll}0.224983 & -0.00898 \\ -0.00898 & 0.004166\end{array}\right]^{-1}\left(\boldsymbol{Z}-\left[\begin{array}{l}\hat{Z}_{1} \\ \hat{Z}_{2}\end{array}\right]\right) \leq 0.005$,

半长轴的折减系数取 0.55 轴长折减为 0.287 , 方 向为 $\boldsymbol{u}_{1}=\left[\begin{array}{ll}-0.99918 & 0.040546\end{array}\right]^{\mathrm{T}}$, 半短轴的折减系数取 0.4 , 轴长折减为 0.0372 , 方向为 $\boldsymbol{u}_{2}=[-0.99918$ $-0.040546]^{\mathrm{T}}$.

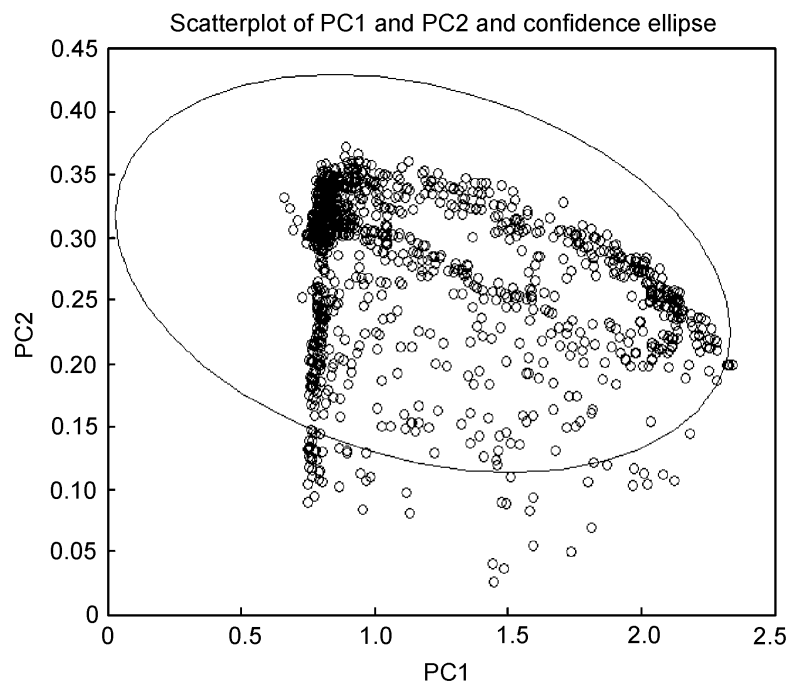

图 4 整体观察值的控制域

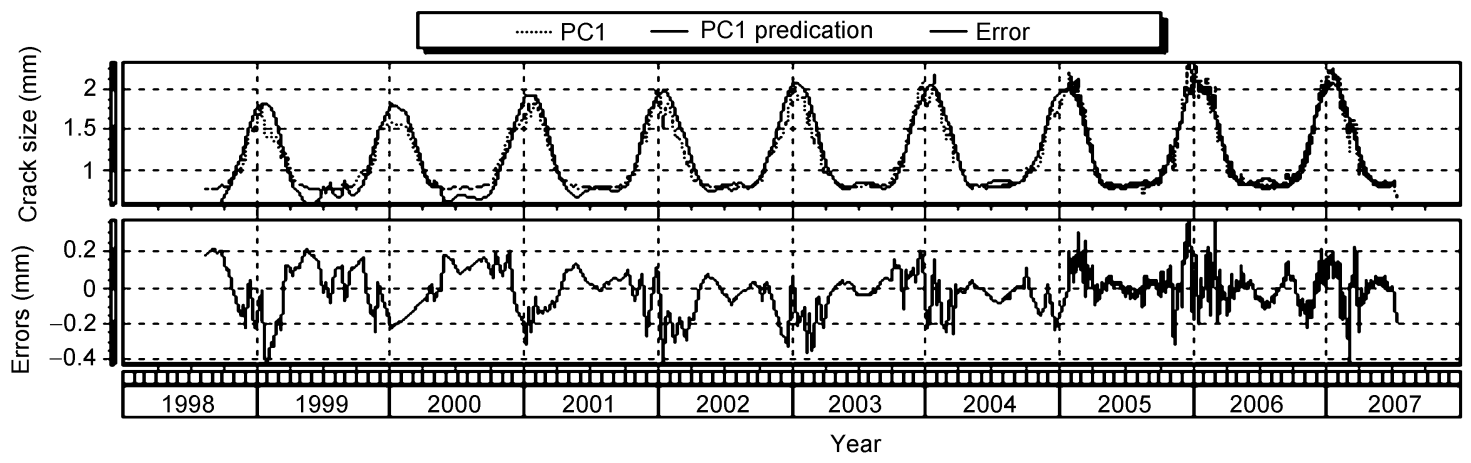

图 5 PC1 实测拟合过程线 


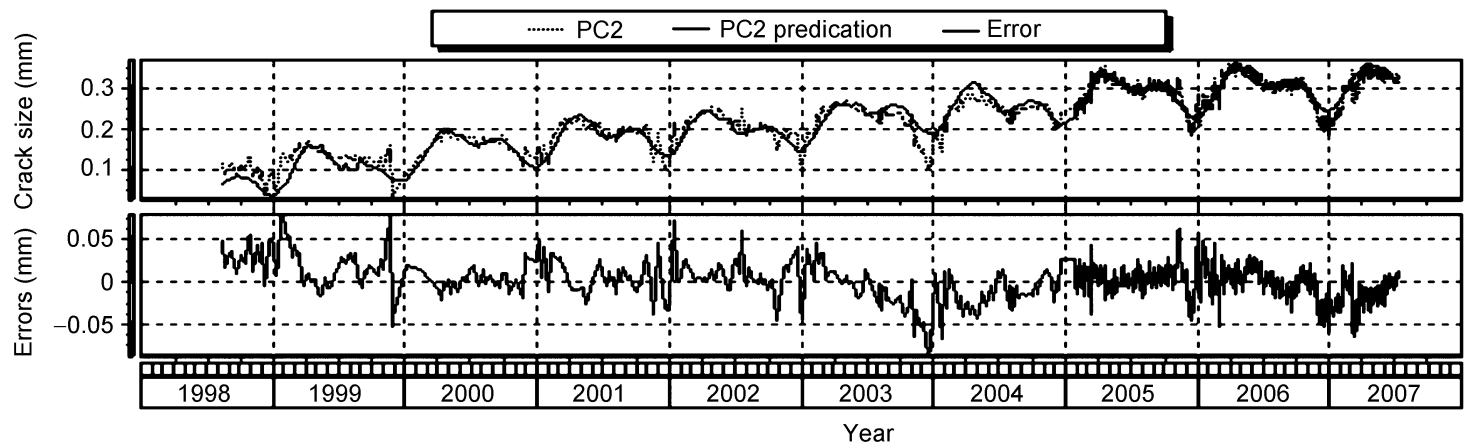

图 6 PC2 实测拟合过程线

\section{表 4 PC1 和 PC2 的 HST 模型参数汇总}

\begin{tabular}{cccccccccccccccc}
\hline & 常数 & $a_{1}$ & $a_{2}$ & $a_{3}$ & $a_{4}$ & $a_{5}$ & $a_{6}$ & $a_{7}$ & $a_{8}$ & $c_{1}$ & 0.373 & -0.320 & 0.032 & $-2.206 \times 10^{-7}$ \\
\hline PC1 & -3.142 & -2.599 & 9.930 & -14.223 & 6.962 & 0.241 & 0.576 & 0.373 \\
PC2 & -1.462 & 0 & 0.221 & -0.750 & 0.524 & 0.012 & -0.040 & -0.024 & 0.049 & 0.011 & $-5.601 \times 10^{-8}$ \\
\hline
\end{tabular}

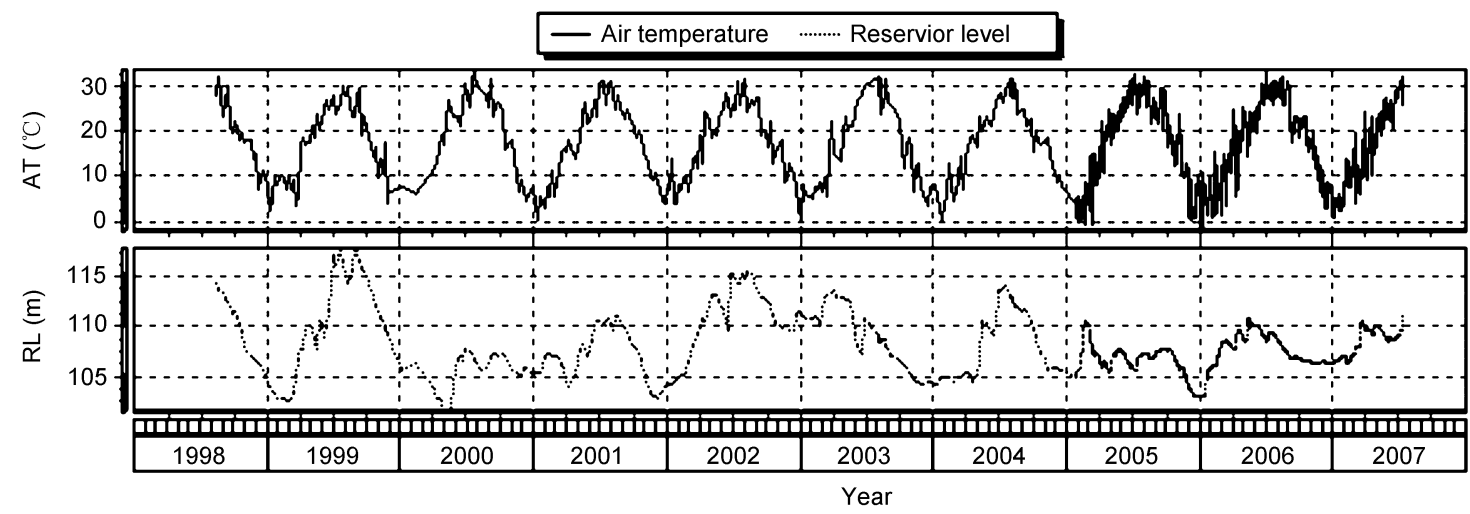

图 7 环境温度和库水位过程线

选取 2007 年主成分峰值附近的预测值绘制观测 值控制域(图 8 所示), 控制域完全包括了观测值, 说 明裂缝的性态未发生转异, 大坝在当前荷载组合下 可以正常运行.

\section{4 结论}

本文深入分析了大坝安全监测系统采集的海量 数据中存在的数据冗余和测量噪声现象, 指出传统 的单效应量分析割裂大多数效应量之间的相关关系, 不仅造成分析的滞后和延迟，还导致了虚假警情随 效应量的规模呈现指数级增长, 影响分析的效率和 限制其应用泛围. 在数据分析和监控工作中引入主

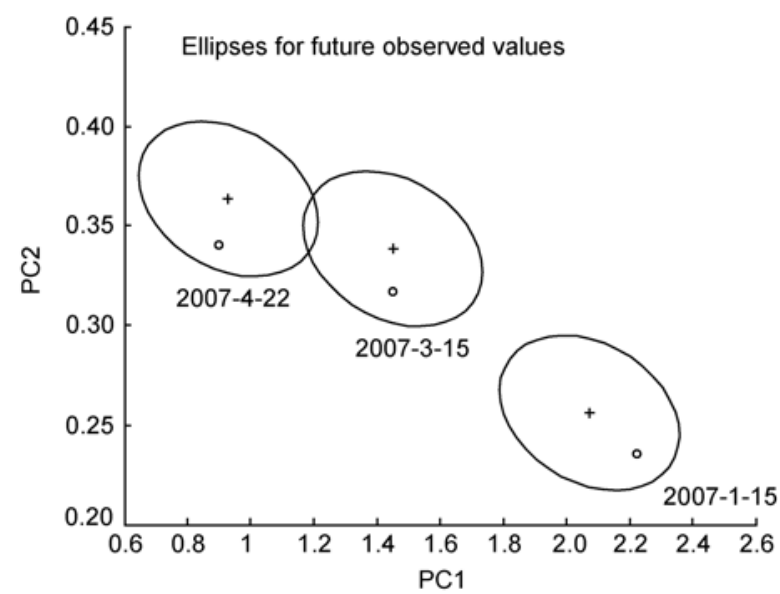

图 8 未来观测值的置信椭圆 
成分分析的原理和方法, 并通过工程实例说明可以 有效地解决上述问题, 主要表现如下.

1) 数据缩减. 主成分分析利用效应量之间的相 关关系提取主成分，因此对原始观测资料无新增的 特殊要求, 并可以有效的减少数据冗余, 重构后的主 成分一般不超过 $4 \sim 5$ 个, 数据缩减率可以 $60 \%$ 以上.

2) 噪声过滤. 主成分可以在较大的程度上分离 信号与噪声, 一般均在 $90 \%$ 以上, 可以处理大多数的
异常数据, 有效地减少粗差处理的工作量.

3) 多效应量分析和监控. 主成分分析可以对多 效应量进行分析，可以适用于不同坝型的各种存在 相关关系的观测项目. 该方法利用多维的置信椭球 对多效应量进行监控, 改变了传统的单效应分析模 式，提高了数据分析、结构安全监控的效率. 同时虚 假警情也能得到有效的控制，拓展了原有监控系统 的应用范围。

\section{参考文献}

1 National Research Council. Safety of Existing Dams Evaluation and Improvement. Washington D.C.: National Academy Press, 1983. 4-40

2 SDJ336-89. 混凝土大坝安全监控规范. 1990. 1255-1256

3 Dibiagio E. Monitoring of dams and their foundations. In: 20th International Commission on Large Dams. Beijing: ICOLD, 2000

4 任若恩, 王惠文. 多元统计数据分析一一理论、方法、实例. 北京: 国防工业出版社, 1997.92

5 Behrouz A N. Multivariate Statistical Analysis of Monitoring Data for Concrete Dams. Canada Montreal: McGill University, 2002. 97-102

$6 \mathrm{Li} \mathrm{X} \mathrm{H,} \mathrm{Xu} \mathrm{H} \mathrm{Z,} \mathrm{Gu} \mathrm{C} \mathrm{S,} \mathrm{et} \mathrm{al.} \mathrm{Application} \mathrm{of} \mathrm{principal} \mathrm{component} \mathrm{fuzzy} \mathrm{neural} \mathrm{network} \mathrm{model} \mathrm{to} \mathrm{analysis} \mathrm{of} \mathrm{observation} \mathrm{data} \mathrm{of} \mathrm{dams} \mathrm{(in}$ Chinese). Dam Obs Geotech Test, 2001. 14-16

7 Liu C D. Analysis Method of Multi-Factor Weightings of Dam Safety Evaluation and its Application (in Chinese). Nanjing: Hohai University, 2004. 20-22

8 Chen L. Mechanics performance with parameters changing in space \& monitoring models of roller compacted concrete dam (in Chinese). Dissertation of Masteral Degree. Nanjing: Hohai University, 2006. 74-76

9 Rencher A C. Multivariate Statistical Inference and Applications. New York: John Wiley \& Sons, 1998. 119-126

10 Martens H, Nase T. Multivariate Calibration. New York: John Wiley \& Sons, 1989. 141-142

11 Bonelli S, Royet P. Delayed response analysis of dam monitoring data. In: ICOLD European Symposium. Lisse, 2001. 91—99 